\title{
Product Conceptual Design Method Based on Intuitionistic Fuzzy Binary Semantics Group Decision Making
}

\author{
Xiaoguang Zhou*, Yongpeng Wu, Vendula Polochova \\ Donlinks School of Economics and Management, University of Science and Technology Beijing, Beijing, China \\ Email: *xiaoguang@ustb.edu.cn, 1824336152@qq.com, vendulapolochova@163.com
}

How to cite this paper: Zhou, X.G., Wu, Y.P. and Polochova, V. (2019) Product Conceptual Design Method Based on Intuitionistic Fuzzy Binary Semantics Group Decision Making. Journal of Service Science and Management, 12, 742-754.

https://doi.org/10.4236/jssm.2019.126050

Received: September 6, 2019

Accepted: October 6, 2019

Published: October 9, 2019

Copyright () 2019 by author(s) and Scientific Research Publishing Inc. This work is licensed under the Creative Commons Attribution International License (CC BY 4.0). http://creativecommons.org/licenses/by/4.0/

\begin{abstract}
Faced with a fiercely competitive market environment, enterprises are investing huge resources, such as human resource, capital and material resources to research and develop new products. The product conceptual design determines the quality, cost and reliability of the final product and is considered to be the most important stage in the product life cycle. Considering the ambiguity of information in the product conceptual design process and the interactivity of experts in selecting conceptual products, a product conceptual design method based on intuitionistic fuzzy binary semantics group decision making is proposed, and a case is used to illustrate the proposed method.
\end{abstract}

\section{Keywords}

Product Conceptual Design, Group Decision Making, Binary Semantics, Conceptual Product, Intuitionistic Fuzzy Set

\section{Introduction}

New product development is a crucial process in maintaining a company's competitive position and succeeding in dynamic markets. Conceptual design plays an important role in development of new products and redesign of existing products. Brunetti and Golob [1] proposed an approach incorporates a feature-based representation scheme for capturing product semantics in the conceptual design phase and links early design with part and assembly modelling. Tay and $\mathrm{Gu}$ [2] presented a function-based model for conceptual design. To close the loop for supporting designers in generating design concepts flexibly, fast, and easily, an ontology-based approach for knowledge management that 
works along with the graphical modeling tool is explored [3]. Focusing on the solution search phase, Liikkanen and Perttula [4] analysed explicit and implicit problem decomposition techniques and integrated them into a descriptive cognitive model. Gehin et al. [5] introduced a method to support designers in the definition of the product lifecycle scenario when designing the elements of the structure of the product, and the proposed method was illustrated via a fridge case study. Li et al. [6] proposed a conceptual design process model to aid multi-stage innovation of product design based on the integration of the essential rules of the axiomatic design model, function-behaviour-structure model, and the guideline of functional creative thinking logics. To draw up a framework proposal for integrated materials and process selection in product design, Albiñana and Vila [7] defined a workflow based on the relations among the parameters of the whole life cycle. There are many open source applications in Computer Aided Design; however there is no on-line environment; hence Suaza et al. [8] presented Web 2.0 online technology for conceptual design. With the development of internet technology, it becomes possible to combine designers from different fields into one team to support product design. A distributed collaborative product design environment is investigated to support top-down process-oriented product design [9]. The use of digital sculpting software is proposed as a way of producing $3 \mathrm{D}$ sketches in the process of conceptual product design [10]. To enhance the designers' awareness during the conceptual design of a product service system, Bertoni [11] presented the testing activity of a color-coded 3D visualization approach. Case-based reasoning is a promising methodology for assisting conceptual product design. Li and Xie [12] proposed a modularized generic product model for managing one-of-a-kind production product families. Weiss and Hari [13] improved the systematic method for conceptual design of a new product introduced by Pahl and Beitz in 1977. Gani and $\mathrm{Ng}$ [14] provided an overview of product design, including four product types: molecular products, formulated products, devices and functional products.

The use of platform-based product family design of assembled products has been reconceptualised into a framework of platform-based design of non-assembled products for the process industries [15]. Szejka et al. [16] developed a semantic reconciliation view to support the interoperable information relationships in product design and manufacturing. Bourgeois-Bougrine et al. [17] discussed the effectiveness of the creativity training to improve the students' confidence, as well as the fit between the tools used and the conceptual design challenge, the phase of the creative process, and individual preferences. Based on axiomatic design and theory of solving inventive problems, Ko [18] presented a novel hybrid-compact design matrix by integrating problem analysis and idea generation approaches into the conceptual design stage for new product innovation. Filho et al. [19] explored the state of art about smart products and designed a self-aware smart product in a smart factory production environment. Relich and Pawlewski [20] developed a case-based reasoning approach towards using neural networks 
to estimate the cost of new product development in one-of-a-kind production companies. Zhang et al. [21] developed a conceptual model for manufacturers to redesign product and identified additive manufacturing process adoption opportunities. He et al. [22] devoted to conceptual design evaluation for product environmental footprint using unascertained measure model. Jiang et al. [23] proposed the concept of the performance value of the principle solution, and a scheme is improved.

Multi-objective optimization is used for the conceptual design of a new industrial process for the production of poly dimethyl ethers [24]. Ilgin et al. [25] presented over 190 multi-criteria decision making studies in environmentally conscious manufacturing and product recovery. Bracke et al. [26] introduced a concept, how to make eco- and sustainability-related decisions within the conceptual design phase of a subsequently product generation, and the decision concept is explained by an automotive engineering example.

The uncertainties due to the subjective evaluations from engineers and customers are not considered in most existing conceptual design approaches. For conceptual design of mechatronics system, various tools like fuzzy delphi method, fuzzy interpretive structural modeling, fuzzy analytical network process and fuzzy quality function deployment can be used [27] [28]. Büyüközkan and Güleryüz [29] proposed a combined intuitionistic fuzzy group decision making model that consists of the intuitionistic fuzzy analytic hierarchy process and intuitionistic fuzzy technique for order preference by similarity to ideal solution methods for effectively evaluating product development partners. A systematic decision making approach is developed for product conceptual design based on fuzzy morphological matrix to quantitatively evaluate function solution principles using knowledge and preferences of engineers and customers with subjective uncertainties [30].

The concept of intuitionistic fuzzy set (IFS) was presented by Atanassov [31], which is the generalization of fuzzy set. As an IFS assigns to each element a membership degree, a non-membership degree and a hesitation degree, therefore, the IFS is more expressive in dealing with uncertain and vague information than fuzzy set. This paper proposed a conceptual design method based on intuitionistic fuzzy binary semantics group decision making, which is supposed to make the conceptual design process more scientific and reliable.

\section{Basic Definitions and Operation Rules}

Combining the advantages of intuitionistic fuzzy set and binary semantics, intuitionistic fuzzy binary semantics can express the uncertainty and fuzziness in the decision-making process well. The definitions and operation rules of intuitionistic fuzzy binary semantics are given as follows.

Definition 1 Let $S=\left\{s_{i} \mid i=1,2, \cdots, g\right\}$ be a language term set, $\tilde{S}$ is the intuitionistic fuzzy binary semantic set generated by $S$, and

$\tilde{S}=\left\{\left(\mu s_{i}, v s_{i+1}\right), \mu, v \in[0,1], \mu+v \leq 1, i=1,2, \cdots, g-1\right\}$. Element $\left(\mu s_{i}, v s_{i+1}\right)$ in- 
dicates that the membership degree of a scheme belongs to $s_{i}$ is $\mu$, and the membership degree belongs to $s_{i+1}$ is $V$.

Definition 2 Let $\tilde{S}=\left\{\left(\mu s_{i}, v s_{i+1}\right), \mu, v \in[0,1], \mu+v \leq 1, i=1,2, \cdots, g-1\right\}$ be an intuitionistic fuzzy binary semantic set. We can define a mapping $f$, and which can convert $\tilde{S}$ into corresponding interval values.

$$
f\left(\mu s_{i}, v s_{i+1}\right)=[(1-b)+i, a+i]
$$

where, $a=1-\mu, \quad b=1-v$. The subscript $1,2, \cdots, g$ represents the corresponding language variables. Then formula (1) can be further rewritten as:

$$
f\left(\mu s_{i}, v s_{i+1}\right)=[v+i, 1-\mu+i]=\left[\mu^{L}, \mu^{R}\right]
$$

where, $u^{L}=v+i, u^{R}=1-\mu+i$.

During the process of group decision making, it is necessary to aggregate the evaluation information on the basis of the weight of each indicator, and also need to summarize the opinions of experts. Therefore, the intuitionistic fuzzy binary semantics should be integrated.

Definition 3 Let $\tilde{S}=\left(\left(\mu_{1} s_{1}, v_{1} s_{2}\right),\left(\mu_{2} s_{2}, v_{2} s_{3}\right), \cdots,\left(\mu_{n} s_{n}, v_{n} s_{n+1}\right)\right)$ be an intuitionistic fuzzy binary semantic set, and $W=\left\{\omega_{1}, \omega_{2}, \cdots, \omega_{n}\right\}$ is the corresponding weight vector, where $\omega_{i} \in[0,1]$, and $\sum_{i=1}^{n} \omega_{i}=1$. The weighted average set of intuitionistic fuzzy binary semantics is:

$$
\operatorname{TWA}(\tilde{s})=\sum_{i=1}^{n}\left(\omega_{i} f\left(\mu s_{i}, v s_{i+1}\right)\right)=\sum_{i=1}^{n}\left[\omega_{i} \mu^{L}, \omega_{i} \mu^{R}\right]
$$

The membership degrees of the intuitionistic fuzzy binary semantics can be converted into interval values by formula (1). Hence the operation rules of interval values can be adopted. Inspired by the reference [32], the distance between two intuitionistic fuzzy binary semantic intervals can be defined.

Definition 4 If the intuitionistic fuzzy binary semantic sets $\tilde{R}^{k}=\left(\tilde{r}_{1}, \tilde{r}_{2}, \cdots, \tilde{r}_{n}\right)$ generated from the language terms set $S=\left\{s_{i} \mid i=1,2, \cdots, g\right\}$, where $k=1,2$, then the distance between the two sets $\tilde{R}^{1}$ and $\tilde{R}^{2}$ is:

$$
d\left(\tilde{R}^{1}, \tilde{R}^{2}\right)=\frac{1}{n}\left(\sum_{i=1, j=1}^{n}\left(\frac{1}{2}\left(\left|\mu^{L}\left(\tilde{r}_{i}\right)-\mu^{L}\left(\tilde{r}_{j}\right)\right|\right)^{\theta}+\frac{1}{2}\left(\left|\mu^{R}\left(\tilde{r}_{i}\right)-\mu^{R}\left(\tilde{r}_{j}\right)\right|\right)^{\theta}\right)^{\frac{1}{\theta}}\right)
$$

The reverse distance between $\tilde{R}^{1}$ and $\tilde{R}^{2}$ can be defined as:

$$
T\left(\tilde{R}^{1}, \tilde{R}^{2}\right)=g-d\left(\tilde{R}^{1}, \tilde{R}^{2}\right)
$$

For formula (5), the larger the reverse distance $T$, the closer the set $\tilde{R}^{1}$ and $\tilde{R}^{2}$ are, and the more similar the two sets are. For $\tilde{R}^{1}$ and $\tilde{R}^{2}$, the following properties hold.

1) $0 \leq T\left(\tilde{R}^{1}, \tilde{R}^{2}\right) \leq g$;

2) $T\left(\tilde{R}^{1}, \tilde{R}^{2}\right)=T\left(\tilde{R}^{2}, \tilde{R}^{1}\right)$;

3) $T\left(\tilde{R}^{1}, \tilde{R}^{2}\right)=g$, if and only if $\tilde{R}^{1}$ and $\tilde{R}^{2}$ are completely similar;

4) $T\left(\tilde{R}^{1}, \tilde{R}^{2}\right)=0$, if and only if $\tilde{R}^{1}$ and $\tilde{R}^{2}$ are completely different.

Since the proof is simple, hence it is ignored here.

How to determine the weights of decision makers during the group decision 
process? The technique for order preference by similarity to ideal solution (TOPSIS) method is adopted in this paper. The basic principle is to calculate the distances between different opinions according to formulas (4) and (5). If the decision maker's opinion differs greatly from the ideal optimal opinion, then a smaller weight is assigned to the decision maker. Otherwise, a larger weight is assigned.

Definition 5 The weight of the $k$ th decision maker can be determined as,

$$
\lambda_{k}=\frac{T\left(\tilde{R}^{k}, \tilde{R}^{+}\right)+d\left(\tilde{R}^{k}, \tilde{R}^{-}\right)}{\sum_{k=1}^{l}\left[T\left(\tilde{R}^{k}, \tilde{R}^{+}\right)+d\left(\tilde{R}^{k}, \tilde{R}^{-}\right)\right]}, k=1,2, \cdots, l .
$$

where, $l$ indicates the number of decision makers; $\lambda_{k}$ represents the weight of the $k$ th decision maker; $T\left(\tilde{R}^{k}, \tilde{R}^{+}\right)$denotes the inverse distance between the opinion of the $k$ th decision maker and the ideal optimal opinion; $d\left(\tilde{R}^{k}, \tilde{R}^{-}\right)$ indicates the distance between the opinion of the $k$ th decision maker and the ideal worst opinion; $\tilde{R}^{+}$is the ideal optimal opinion and $\tilde{R}^{-}$is the ideal worst opinion. The distance and the reverse distance are simultaneously considered because for a cell in a large interval, the cell closest to the left border is not necessarily the farthest from the right border.

In practice, the selection of a conceptual product is generally based on the comprehensive optimal principle, that is, the combination of all aspects of indicators to choose the best comprehensive performance. In this paper, the idea of TOPSIS method is adopted, that is, the conceptual product is selected according to the reverse distance between each alternative and the ideal optimal scheme and also the distance from the ideal worst one. The alternative closest to the ideal optimal solution and the farthest from the ideal worst solution is the best solution.

Definition 6 There are two scheme $X_{1}$ and $X_{2}$, if

$$
T\left(X_{1}, \tilde{R}^{+}\right)+d\left(X_{1}, \tilde{R}^{-}\right)>T\left(X_{2}, \tilde{R}^{+}\right)+d\left(X_{2}, \tilde{R}^{-}\right)
$$

Then, the scheme $X_{1}$ is superior to the scheme $X_{2}$.

\section{Group Decision Making Process Based on Intuitionistic Fuzzy Binary Semantics}

Let $X=\left\{X_{1}, X_{2}, \cdots, X_{m}\right\}$ be the set of alternative conceptual products, $C=\left\{C_{1}, C_{2}, \cdots, C_{n}\right\}$ indicates the evaluation indicators, $C_{j}$ denotes the $t$ th indicator, $j=1,2, \cdots, n$. Suppose $l$ decision makers are invited to evaluate conceptual products, and $E_{k}$ indicates the evaluation opinion of the $k$ th decision maker, marked as $E=\left\{E_{1}, E_{2}, \cdots, E_{l}\right\} . \lambda_{k}$ represents the weight of the $k$ th decision maker, where $\sum_{k=1}^{l} \lambda_{k}=1, k=1,2, \cdots, l$, and $\lambda_{k}$ is a normalized weight vector. The evaluation opinions of decision makers about the indicators of the alternative conceptual products are expressed by intuitionistic fuzzy binary semantics. The framework of intuitionistic fuzzy binary semantic group decision process is shown in Figure 1. 


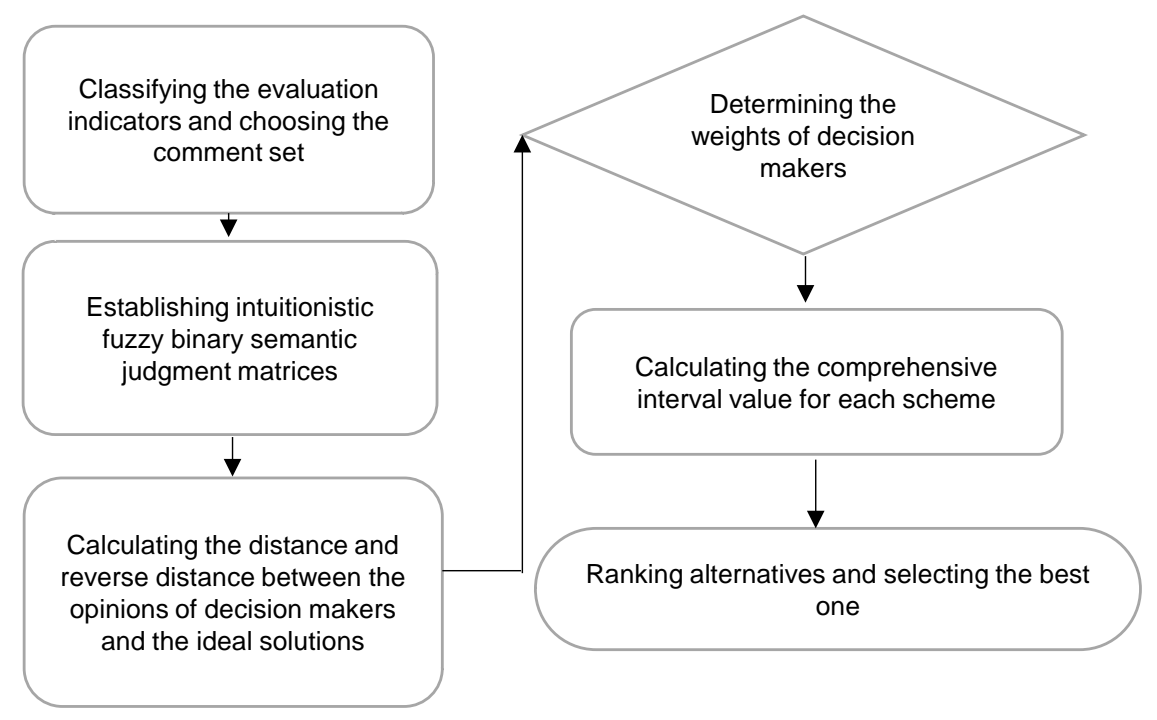

Figure 1. Intuitionistic fuzzy binary semantic group decision process.

The optimal conceptual product selection process is as follows.

Step 1: According to the attribute types of evaluation indices, the corresponding comment sets are selected. The linguistic variables in the comment sets represent the evaluation opinions of decision makers. For instance, a five-level comment set is defined as below:

For benefit-based indicators: the comment set is $\left\{s_{1}\right.$ (very low), $s_{2}$ (low), $s_{3}$ (medium), $s_{4}$ (high), $s_{5}$ (very high)\}. For cost-based indicators: the comment set is $\left\{s_{1}\right.$ (very high), $s_{2}$ (high), $s_{3}$ (medium), $s_{4}$ (low), $s_{5}$ (very low) $\}$.

It should be pointed out that different levels of comment sets, such as seven-level or nine-level, can be used according to actual conditions.

Step 2: The decision makers evaluate alternative conceptual products and thus the corresponding judgment matrices are formed. Assume that there are $C_{j}(j=1,2, \cdots, n)$ indicators of $X_{i}(i=1,2, \cdots, m)$ conceptual product need to be evaluated by $E_{k}(k=1,2, \cdots, l)$ decision makers, and the evaluation opinion $\tilde{r}_{i j}^{k}$ is denoted by intuitionistic fuzzy binary semantics, that is,

$\tilde{r}_{i j}^{k}=\left(\mu s_{i}\left(x_{i j}\right), v s_{i+1}\left(x_{i j}\right)\right), x_{i j}$ represents the $C_{j}$ attribute of the product $X_{i}$. The decision opinion of each decision maker constitutes an intuitionistic fuzzy binary semantic decision matrix $\tilde{R}^{k}=\left(\tilde{r}_{i j}^{k}\right)_{m \times n}$.

$$
\tilde{R}^{k}=\left[\begin{array}{cccc}
\tilde{r}_{11}^{k} & \tilde{r}_{12}^{k} & \cdots & \tilde{r}_{m}^{k} \\
\tilde{r}_{21}^{k} & \tilde{r}_{22}^{k} & \cdots & \tilde{r}_{2 m}^{k} \\
\vdots & \vdots & \ddots & \vdots \\
\tilde{r}_{n 1}^{k} & \tilde{r}_{n 2}^{k} & \cdots & \tilde{r}_{n m}^{k}
\end{array}\right]
$$

The intuitionistic fuzzy binary semantic decision matrix can be rewritten according to formulas (1) and (2), and the corresponding interval evaluation values are obtained. The opinions of decision makers are assembled according to formula (3).

Step 3: According to formulas (4) and (5), the distances and reverse distances 
between the opinions of decision makers and the virtual optimal/worst scheme are calculated.

Step 4: According to formula (6), the weights of decision makers can be achieved. The formula (3) is used to aggregate the opinions of the various decision makers, and the evaluation interval values of alternative conceptual products are acquired.

Step 5: Select the best conceptual product on the basis of formula (7).

\section{Product Conceptual Design Based on Intuitionistic Fuzzy Binary Semantics Group Decision Making Method}

Assume that there are four types of conceptual products have been selected through the process of product conceptual design, as shown in Table 1.

A total of four decision makers further evaluated the four kinds of alternative conceptual products: product function $C_{1}$, product structure $C_{2}$, operation behavior $C_{3}$, and product cost $C_{4}$. The weights of indicators can be acquired by methods such as analytic network process, entropy method, and principal component analysis. This paper does not focus on the weights of indicators. Suppose these four indicators have the same weights:

$$
W^{\mathrm{T}}=\left(\omega_{1}, \omega_{2}, \omega_{3}, \omega_{4}\right)^{\mathrm{T}}=(0.25,0.25,0.25,0.25)^{\mathrm{T}}
$$

1) Determining the comment sets, as shown in Table 2.

2) Assume that the evaluation information given by the four decision makers are as follows.

$$
\begin{aligned}
\tilde{R}^{1} & =\left(\begin{array}{llll}
0.8 s_{4}, 0.1 s_{5} & 0.3 s_{3}, 0.4 s_{4} & 0.6 s_{2}, 0.2 s_{3} & 0.2 s_{2}, 0.5 s_{3} \\
0.6 s_{3}, 0.2 s_{4} & 0.7 s_{4}, 0.2 s_{5} & 0.5 s_{3}, 0.3 s_{4} & 0.2 s_{2}, 0.6 s_{3} \\
0.8 s_{2}, 0.2 s_{3} & 0.8 s_{2}, 0.2 s_{3} & 0.7 s_{2}, 0.2 s_{3} & 0.2 s_{4}, 0.6 s_{5} \\
0.3 s_{3}, 0.4 s_{4} & 0.3 s_{3}, 0.6 s_{4} & 0.5 s_{3}, 0.3 s_{4} & 0.2 s_{3}, 0.7 s_{4}
\end{array}\right) \\
\tilde{R}^{2} & =\left(\begin{array}{lllll}
0.2 s_{4}, 0.5 s_{5} & 0.2 s_{3}, 0.7 s_{4} & 0.6 s_{2}, 0.2 s_{3} & 0.6 s_{2}, 0.3 s_{3} \\
0.3 s_{2}, 0.6 s_{3} & 0.3 s_{4}, 0.7 s_{5} & 0.5 s_{3}, 0.2 s_{4} & 0.5 s_{2}, 0.2 s_{3} \\
0.3 s_{2}, 0.6 s_{3} & 0.7 s_{2}, 0.2 s_{3} & 0.6 s_{2}, 0.3 s_{3} & 0.8 s_{4}, 0.2 s_{5} \\
0.3 s_{3}, 0.5 s_{4} & 0.4 s_{3}, 0.5 s_{4} & 0.4 s_{3}, 0.5 s_{4} & 0.2 s_{3}, 0.5 s_{4}
\end{array}\right) \\
\tilde{R}^{3} & =\left(\begin{array}{llll}
0.2 s_{4}, 0.5 s_{5} & 0.2 s_{3}, 0.8 s_{4} & 0.5 s_{2}, 0.2 s_{3} & 0.7 s_{3}, 0.2 s_{4} \\
0.5 s_{2}, 0.3 s_{3} & 0.5 s_{4}, 0.3 s_{5} & 0.8 s_{3}, 0.2 s_{4} & 0.3 s_{2}, 0.5 s_{3} \\
0.3 s_{2}, 0.6 s_{3} & 0.8 s_{2}, 0.2 s_{3} & 0.6 s_{2}, 0.4 s_{3} & 0.6 s_{4}, 0.2 s_{5} \\
0.3 s_{3}, 0.5 s_{4} & 0.4 s_{3}, 0.5 s_{4} & 0.4 s_{3}, 0.5 s_{4} & 0.3 s_{3}, 0.5 s_{4}
\end{array}\right) \\
\tilde{R}^{4}= & \left(\begin{array}{llll}
0.3 s_{4}, 0.5 s_{5} & 0.7 s_{3}, 0.1 s_{4} & 0.2 s_{2}, 0.6 s_{3} & 0.7 s_{2}, 0.1 s_{3} \\
0.4 s_{3}, 0.4 s_{4} & 0.5 s_{4}, 0.4 s_{5} & 0.4 s_{2}, 0.5 s_{3} & 0.2 s_{2}, 0.6 s_{3} \\
0.6 s_{2}, 0.1 s_{3} & 0.5 s_{2}, 0.4 s_{3} & 0.4 s_{2}, 0.4 s_{3} & 0.8 s_{4}, 0.2 s_{5} \\
0.3 s_{3}, 0.5 s_{4} & 0.6 s_{3}, 0.2 s_{4} & 0.8 s_{3}, 0.1 s_{4} & 0.4 s_{3}, 0.4 s_{4}
\end{array}\right)
\end{aligned}
$$

where, each row denotes the opinions of decision makers about a conceptual product, and each column represents the opinions of decision makers about an indicator. 
Table 1. Alternative conceptual products.

\begin{tabular}{cc}
\hline Product Category & Product Characteristics \\
\hline Class $A$ & Focus on functional design and improving functional innovation \\
Class $B$ & Focus on improving product structure performance \\
Class $C$ & Focus on cost savings \\
Class $D$ & Not specializing, but considering many aspects \\
\hline
\end{tabular}

Table 2. Evaluation indicators and comment sets.

\begin{tabular}{lll}
\hline Evaluation indicators & Indicator type & Comment set \\
\hline Product function $C_{1}$ & Income type & $\begin{array}{l}S=\left\{s_{1} \text { (very low), } s_{2} \text { (low), } s_{3} \text { (medium), } s_{4} \text { (high), } s_{5} \text { (very }\right. \\
\text { high) }\}\end{array}$ \\
Product structure $C_{2}$ & Income type & $\begin{array}{l}S=\left\{s_{1} \text { (very low), } s_{2} \text { (low), } s_{3} \text { (medium), } s_{4} \text { (high), } s_{5} \text { (very }\right. \\
\text { high) }\}\end{array}$ \\
Operation behavior $C_{3}$ Income type & $\begin{array}{l}S=\left\{s_{1} \text { (very low), } s_{2} \text { (low), } s_{3} \text { (medium), } s_{4} \text { (high), } s_{5} \text { (very }\right. \\
\text { high) }\}\end{array}$ \\
Product cost $C_{4}$ & Cost type & $\begin{array}{l}S=\left\{s_{1} \text { (very high), } s_{2} \text { (high), } s_{3} \text { (medium), } s_{4} \text { (low), } s_{5} \text { (very }\right. \\
\text { low) }\}\end{array}$ \\
\hline
\end{tabular}

According to formulas (1) and (2), the above intuitionistic fuzzy binary semantic sets can be converted into:

$$
\begin{gathered}
f\left(\tilde{R}^{1}\right)=\left(\begin{array}{llll}
{[4.1,4.2]} & {[3.4,3.7]} & {[2.2,2.4]} & {[2.5,2.8]} \\
{[3.2,3.4]} & {[4.2,4.3]} & {[3.3,3.5]} & {[2.6,2.8]} \\
{[2.2,2.2]} & {[2.2,2.2]} & {[2.2,2.3]} & {[4.6,4.8]} \\
{[3.4,3.7]} & {[3.6,3.7]} & {[3.3,3.5]} & {[3.7,3.8]}
\end{array}\right) \\
f\left(\tilde{R}^{2}\right)=\left(\begin{array}{llll}
{[4.5,4.8]} & {[3.7,3.8]} & {[2.2,2.4]} & {[2.3,2.4]} \\
{[2.6,2.7]} & {[4.7,4.7]} & {[3.2,3.5]} & {[2.2,2.5]} \\
{[2.6,2.7]} & {[2.2,2.3]} & {[2.3,2.4]} & {[4.2,4.2]} \\
{[3.5,3.7]} & {[3.5,3.6]} & {[3.5,3.6]} & {[3.5,3.8]}
\end{array}\right) \\
f\left(\tilde{R}^{3}\right)=\left(\begin{array}{llll}
{[4.5,4.8]} & {[3.8,3.8]} & {[2.2,2.5]} & {[3.2,3.3]} \\
{[2.3,2.5]} & {[4.3,4.5]} & {[3.2,3.2]} & {[2.5,2.7]} \\
{[2.6,2.7]} & {[2.2,2.2]} & {[2.4,2.4]} & {[4.2,4.4]} \\
{[3.5,3.7]} & {[3.5,3.6]} & {[3.5,3.6]} & {[3.5,3.7]}
\end{array}\right) \\
f\left(\tilde{R}^{4}\right)=\left(\begin{array}{llll}
{[4.5,4.7]} & {[3.1,3.3]} & {[2.6,2.8]} & {[2.1,2.3]} \\
{[3.4,3.6]} & {[4.4,4.5]} & {[2.5,2.6]} & {[2.6,2.8]} \\
{[2.1,2.4]} & {[2.4,2.5]} & {[2.4,2.6]} & {[4.2,4.2]} \\
{[3.5,3.7]} & {[3.2,3.4]} & {[3.1,3.2]} & {[3.4,3.6]}
\end{array}\right)
\end{gathered}
$$

According to formula (3), the information of evaluation indices are assembled, and the comprehensive evaluation intervals of the four types of conceptual products are obtained:

$$
f\left(\tilde{R}^{1}\right)=\left(\begin{array}{l}
{[3.05,3.28]} \\
{[3.33,3.50]} \\
{[2.80,2.88]} \\
{[3.50,3.68]}
\end{array}\right),
$$




$$
\begin{aligned}
& f\left(\tilde{R}^{2}\right)=\left(\begin{array}{l}
{[3.18,3.35]} \\
{[3.18,3.35]} \\
{[2.83,2.90]} \\
{[3.50,3.68]}
\end{array}\right), \\
& f\left(\tilde{R}^{3}\right)=\left(\begin{array}{l}
{[3.43,3.60]} \\
{[3.08,3.23]} \\
{[2.85,2.93]} \\
{[3.50,3.65]}
\end{array}\right), \\
& f\left(\tilde{R}^{4}\right)=\left(\begin{array}{l}
{[3.08,3.28]} \\
{[3.23,3.38]} \\
{[2.78,2.93]} \\
{[3.30,3.48]}
\end{array}\right),
\end{aligned}
$$

The virtual optimal and worst comprehensive evaluation interval values of the four types of conceptual products are:

$$
f\left(\tilde{R}^{+}\right)=\left(\begin{array}{l}
{[5.00,5.00]} \\
{[5.00,5.00]} \\
{[5.00,5.00]} \\
{[5.00,5.00]}
\end{array}\right), \quad f\left(\tilde{R}^{-}\right)=\left(\begin{array}{l}
{[1.00,1.00]} \\
{[1.00,1.00]} \\
{[1.00,1.00]} \\
{[1.00,1.00]}
\end{array}\right) .
$$

1) According to formulas (4) and (5), the distances between each alternative conceptual product and the virtual worst scheme and their reverse distances from the virtual optimal scheme were calculated, where $\theta=2$.

$$
\begin{aligned}
& d\left(\tilde{R}^{1}, \tilde{R}\right)=\left(\begin{array}{l}
{[1.84,2.17]} \\
{[1.59,2.41]} \\
{[2.16,1.84]} \\
{[1.42,2.59]}
\end{array}\right), \\
& d\left(\tilde{R}^{2}, \tilde{R}\right)=\left(\begin{array}{l}
{[1.74,2.26]} \\
{[1.74,2.26]} \\
{[2.14,1.86]} \\
{[1.42,2.59]}
\end{array}\right), \\
& d\left(\tilde{R}^{3}, \tilde{R}\right)=\left(\begin{array}{l}
{[1.49,2.51]} \\
{[1.85,2.15]} \\
{[2.11,1.89]} \\
{[1.43,2.58]}
\end{array}\right), \\
& d\left(\tilde{R}^{4}, \tilde{R}\right)=\left(\begin{array}{l}
{[1.83,2.18]} \\
{[1.70,2.30]} \\
{[2.15,2.85]} \\
{[1.61,2.39]}
\end{array}\right),
\end{aligned}
$$

where, the first column denotes the reverse distances from the optimal scheme, and the second column represents the distances from the worst scheme, and each row indicates a type of conceptual product. Since the maximum distance of 
the semantic set elements is $s_{5}-s_{1}=4$ in this paper, the distance and reverse distance should be ranged between 0 and 4 . That is, for formula (5), it could be modified as:

$$
T\left(\tilde{R}^{1}, \tilde{R}^{2}\right)=4-d\left(\tilde{R}^{1}, \tilde{R}^{2}\right) .
$$

2) Calculating the weights of each decision maker on the basis of formula (6):

$$
\lambda=\left(\begin{array}{llll}
0.24 & 0.25 & 0.27 & 0.24 \\
0.27 & 0.25 & 0.23 & 0.25 \\
0.25 & 0.25 & 0.25 & 0.25 \\
0.26 & 0.26 & 0.25 & 0.23
\end{array}\right) .
$$

In the above matrix, each row denotes the weights of a conceptual product, and each column indicates the weights of a decision maker. That is to say, each alternative conceptual product has its own weight, and each decision maker has different weights for different alternative conceptual products. The reason is that each decision maker has a preference for each candidate conceptual product. This preference is reflected by the evaluation weight for different schemes. The distances between the comprehensive evaluation interval values and the ideal optimal/worst scheme are different. For each type of alternative conceptual product, the process of determining the weight of each decision maker in line with formula (6) is actually the process of gathering opinions of decision makers.

According to formula (3), the final comprehensive evaluation interval values of the four types of alternative conceptual products are obtained:

$$
f(X)=\left(\begin{array}{l}
{[3.19,3.38]} \\
{[3.20,3.37]} \\
{[2.81,2.91]} \\
{[3.45,3.62]}
\end{array}\right)
$$

3) According to formulas (4) and (5), the distances between each candidate conceptual product and the virtual optimal/worst scheme are calculated respectively. The results are as follows:

$$
d(X, \tilde{R})=\left(\begin{array}{l}
{[1.71,2.29]} \\
{[1.72,2.29]} \\
{[2.14,1.86]} \\
{[1.47,2.54]}
\end{array}\right)
$$

where, the first column denotes the distance from the optimal scheme, and the second column represents the distance from the worst scheme. The sum of distance between each alternative conceptual product and the ideal optimal/worst conceptual product can be calculated:

$$
T\left(X, \tilde{R}^{+}\right)+d\left(X, \tilde{R}^{-}\right)=\left(\begin{array}{c}
4.58 \\
4.57 \\
3.72 \\
5.07
\end{array}\right)
$$


According to formula (7), the ranking of the four alternative conceptual products is: $D>A>B>C$, then the kind of conceptual product $D$ is the optimal one. This is in line with the actual situation. Product $D$ is not specialized, however, various factors are comprehensive considered.

\section{Conclusion}

The conceptual product is the prototype of the final product, which is expected to improve people's consumption experience. The conceptual product design is based on the evaluation of the current product and on the assumption of future demand. Therefore, it is important to choose the best conceptual product. This paper proposes a conceptual product selection method based on intuitionistic fuzzy binary semantics group decision making, which can better deal with the fuzziness and uncertainty in the decision making process, and can integrate the opinions of various decision makers to select the optimal conceptual product as well. The case study shows that the proposed method can solve this kind of problem well.

\section{Acknowledgements}

This work was supported by "the National Natural Science Foundation of China (No. 71771023)".

\section{Conflicts of Interest}

The authors declare no conflicts of interest.

\section{References}

[1] Brunetti, G. and Golob, B. (2000) A Feature-Based Approach towards an Integrated Product Model Including Conceptual Design Information. Computer-Aided Design, 32, 877-887. https://doi.org/10.1016/S0010-4485(00)00076-2

[2] Tay, F.E.H. and Gu, J.X. (2002) Product Modeling for Conceptual Design Support. Computer in Industry, 48, 143-155. https://doi.org/10.1016/S0166-3615(02)00014-3

[3] Chang, X., Sahin, A. and Terpenny, J. (2008) An Ontology-Based Support for Product Conceptual Design. Robotics and Computer-Integrated Manufacturing, 24, 755-762. https://doi.org/10.1016/j.rcim.2008.03.004

[4] Liikkanen, L.A. and Perttula, M. (2009) Exploring Problem Decomposition in Conceptual Design among Novice Designers. Design Studies, 30, 38-59. https://doi.org/10.1016/j.destud.2008.07.003

[5] Gehin, A., Zwolinski, P. and Brissaud, D. (2009) Integrated Design of Product Lifecycles-The Fridge Case Study. CIRP Journal of Manufacturing Science and Technology, 1, 214-220. https://doi.org/10.1016/j.cirpj.2009.05.002

[6] Li, W., Li, Y., Wang, J. and Liu, X. (2010) The Process Model to Aid Innovation of Products Conceptual Design. Expert Systems with Applications, 37, 3574-3587. https://doi.org/10.1016/j.eswa.2009.10.034

[7] Albiñana, J.C. and Vila, C. (2012) A Framework for Concurrent Material and Process Selection during Conceptual Product Design Stages. Materials \& Design, 41, 433-446. https://doi.org/10.1016/j.matdes.2012.05.016 
[8] Suaza, R.L., Magal-Royo, T. and Albiñana, B.J. (2012) New Online Services Oriented to the Conceptual Design of Industrial Products through the Application of Web 2.0. Procedia-Social and Behavioral Sciences, 51, 682-686. https://doi.org/10.1016/j.sbspro.2012.08.224

[9] Wu, T., Pei, X., Lu, Y., Chen, C. and Gao, L. (2013) A Distributed Collaborative Product Design Environment Based on Semantic Norm Model and Role-Based Access Control. Journal of Network and Computer Applications, 36, 1431-1440. https://doi.org/10.1016/j.jnca.2013.02.018

[10] Alcaide-Marzal, J., Diego-Más, J.A., Asensio-Cuesta, S. and Piqueras-Fiszman, B. (2013) An Exploratory Study on the Use of Digital Sculpting in Conceptual Product Design. Design Studies, 34, 264-284. https://doi.org/10.1016/j.destud.2012.09.001

[11] Bertoni, A. (2013) Analyzing Product-Service Systems Conceptual Design: The Effect of Color-Coded 3D Representation. Design Studies, 34, 763-793.

https://doi.org/10.1016/j.destud.2013.02.003

[12] Li, B.M. and Xie, S.Q. (2013) Product Similarity Assessment for Conceptual One-of-a-Kind Product Design: A Weight Distribution Approach. Computers in Industry, 64, 720-731. https://doi.org/10.1016/j.compind.2013.04.001

[13] Weiss, M.P. and Hari, A. (2015) Extension of the Pahl \& Beitz Systematic Method for Conceptual Design of a New Product. Procedia CIRP, 36, 254-260. https://doi.org/10.1016/j.procir.2015.03.010

[14] Gani, R. and Ng, K.M. (2015) Product Design-Molecules, Devices, Functional Products, and Formulated Products. Computers \& Chemical Engineering, 81, 70-79. https://doi.org/10.1016/j.compchemeng.2015.04.013

[15] Lager, T. (2017) A Conceptual Framework for Platform-Based Design of Non-Assembled Products. Technovation, 68, 20-34. https://doi.org/10.1016/j.technovation.2017.09.002

[16] Szejka, A.L., Junior, O.C., Panetto, H., Aubry, A. and Loures, E.F.R. (2017) A Semantic Reconciliation View to Support the Interoperable Information Relationships in Product Design and Manufacturing. IFAC-PapersOnLine, 50, 15896-15903. https://doi.org/10.1016/j.ifacol.2017.08.2357

[17] Bourgeois-Bougrine, S., Buisine, S., Vandendriessche, C., Glaveanu, V. and Lubart, T. (2017) Engineering Students' Use of Creativity and Development Tools in Conceptual Product Design: What, When and How? Thinking Skills and Creativity, 24, 104-117. https://doi.org/10.1016/j.tsc.2017.02.016

[18] Ko, Y. (2017) Modeling a Hybrid-Compact Design Matrix for New Product Innovation. Computers \& Industrial Engineering, 107, 345-359. https://doi.org/10.1016/j.cie.2016.04.016

[19] Filho, M.F., Liao, Y., Loures, E.R. and Canciglieri, O. (2017) Self-Aware Smart Products: Systematic Literature Review, Conceptual Design and Prototype Implementation. Procedia Manufacturing, 11, 1471-1480.

https://doi.org/10.1016/j.promfg.2017.07.278

[20] Relich, M. and Pawlewski, P. (2018) A Case-Based Reasoning Approach to Cost Estimation of New Product Development. Neurocomputing, 272, 40-45. https://doi.org/10.1016/j.neucom.2017.05.092

[21] Zhang, H., Nagel, J.K., Al-Qas, A., Gibbons, E. and Lee, J.J. (2018) Additive Manufacturing with Bioinspired Sustainable Product Design: A Conceptual Model. Procedia Manufacturing, 26, 880-891. https://doi.org/10.1016/j.promfg.2018.07.113

[22] He, B., Xiao, J. and Deng, Z. (2018) Product Design Evaluation for Product Envi- 
ronmental Footprint. Journal of Cleaner Production, 172, 3066-3080.

https://doi.org/10.1016/j.jclepro.2017.11.104

[23] Jiang, S., Jing, L., Sun, T., Xu, Q., Peng, X. and Li, J. (2019) A Conceptual Scheme Improvement Approach Based on the Performance Value of the Principle Solution Taking a Coal Mining Machine as a Case Study. Computers in Industry, 105, 17-34. https://doi.org/10.1016/j.compind.2018.10.009

[24] Burger, J. and Hasse, H. (2013) Multi-Objective Optimization Using Reduced Models in Conceptual Design of a Fuel Additive Production Process. Chemical Engineering Science, 99, 118-126. https://doi.org/10.1016/j.ces.2013.05.049

[25] Ilgin, M.A., Gupta, S.M. and Battaïa, O. (2015) Use of MCDM Techniques in Environmentally Conscious Manufacturing and Product Recovery: State of the Art. Journal of Manufacturing Systems, 37, 746-758. https://doi.org/10.1016/j.jmsy.2015.04.010

[26] Bracke, S., Yamada, S., Kinoshita, Y., Inoue, M. and Yamada, T. (2017) Decision Making within the Conceptual Design Phase of Eco-Friendly Products. Procedia Manufacturing, 8, 463-470. https://doi.org/10.1016/j.promfg.2017.02.059

[27] Parameshwaran, R., Baskar, C. and Karthik, T. (2015) An Integrated Framework for Mechatronics Based Product Development in a Fuzzy Environment. Applied Soft Computing, 27, 376-390. https://doi.org/10.1016/j.asoc.2014.11.013

[28] Zhou, X.G., Wang, J.N. and Sun, F. (2018) Product Conceptual Design Based on Intuitionistic Fuzzy Analytic Network Process. Academic Journal of Manufacturing Engineering, 16, 118-127.

[29] Büyüközkan, G. and Güleryüz, S. (2016) A New Integrated Intuitionistic Fuzzy Group Decision Making Approach for Product Development Partner Selection. Computers \& Industrial Engineering, 102, 383-395. https://doi.org/10.1016/j.cie.2016.05.038

[30] Ma, H., Chu, X., Xue, D. and Chen, D. (2017) A Systematic Decision Making Approach for Product Conceptual Design Based on Fuzzy Morphological Matrix. EXpert Systems with Applications, 81, 444-456. https://doi.org/10.1016/j.eswa.2017.03.074

[31] Atanassov, K.T. (1986) Intuitionistic Fuzzy Sets. Fuzzy Sets \& Systems, 20, 87-96. https://doi.org/10.1016/S0165-0114(86)80034-3

[32] Zhang, H. and Yu, L. (2013) New Distance Measures between Intuitionistic Fuzzy Sets and Interval-Valued Fuzzy Sets. Information Sciences, 245, 181-196. https://doi.org/10.1016/j.ins.2013.04.040 